\title{
Investigations of Resolution Limits for Indirect Microwave Holographic Imaging
}

\author{
Okan Yurduseven, ${ }^{1}$ David Smith, ${ }^{1}$ Bryan Livingstone, ${ }^{1}$ Vladimir Schejbal, ${ }^{2}$ Zhonghui You ${ }^{1}$ \\ ${ }^{1}$ Computing, Engineering and Information Sciences, Northumbria University, \\ Newcastle upon Tyne, United Kingdom \\ ${ }^{2}$ Jan Perner Transport Faculty, University of Pardubice, Pardubice, Czech Republic
}

\begin{abstract}
This work describes how indirect holography which has previously been applied to the determination of antenna radiation patterns can be adapted for the imaging of passive objects. It provides details of how complex scattered field values can be obtained in a simple and inexpensive manner from sampled scalar intensity measurements taken over a single scanning aperture. This work uses indirect holographic techniques to image a number of simple objects including a rectangular metallic plate, a small metal plate covered by a dielectric sheet and a small metallic circular annulus. This work demonstrates that good quality images can be reconstructed from simple scalar intensity patterns. It demonstrates that clear outlines can be obtained in particular from reconstructed phase patterns and that good images can be obtained from objects with dimensions of the order of a half wavelength.
\end{abstract}

Keywords: microwave imaging; microwave holography; near-field microwave imaging; image reconstruction

\section{INTRODUCTION}

Indirect holography offers a simple and inexpensive technique for the determination of complex scattered fields using only scalar intensity measurements taken over a single aperture [1-5]. Such an arrangement does not require the use of vector measuring equipment and can be performed using basic power measuring devices. A number of different techniques have been investigated in order to eliminate the requirement for the direct measurement of phase for complex field reconstruction. These include the use of phase retrieval techniques and indirect holographic techniques. Amplitude only measurements, conducted on two surfaces have been used in Refs. 6-9 where the phase is determined by minimization of the corresponding functional. In Refs. 10-12 two probes are used to perform simultaneous measurements on a single surface and the amplitudes are combined to enable phase information to be retrieved. An alternative technique using intensity measurements taken over a single scanned aperture using a radiated reference signal is described in Ref. 1 although this technique is restricted to small angles. A different holographic technique for antenna measurement has been proposed in Refs. 2-5. In this approach antenna far field radiation characteristics and reconstructed aperture fields can be obtained from intensity measurements taken over a single scanning aperture.

This method differs from direct holographic methods in that the complex field is not measured directly but can be reconstructed from a stationary interference pattern, or hologram, recorded by combining the sampled field of the Antenna Under Test with a known reference signal. The remainder of this article is structured as follows, Section II provides a brief outline of indirect holographic techniques, Section III includes measurements taken on a range of simple metal objects to investigate the ability of indirect holography to provide sharp images of these objects with well defined edges, Section IV investigates the ability of this technique to provide good images of metal objects obscured by covering dielectric material, and Section V provides concluding remarks.

\section{INDIRECT MICROWAVE HOLOGRAPHY}

An outline of the technique is given below in Figure 1. The output from the microwave generator is split by the directional coupler to provide one signal to illuminate the object under investigation and a second signal which will form the reference signal.

The first signal is fed directly to a section of open ended waveguide which acts as an illuminating antenna. The signal scattered from the object is picked up by a second section of open ended waveguide. This signal is applied to one of the inputs to the hybrid tee. A phase coherent reference signal, $\operatorname{Er}(\mathrm{x}, \mathrm{y})$, is applied to the second input of the hybrid tee. This reference signal is assumed to have a uniform amplitude and a linearly increasing phase shift which can be applied along the x-direction, along the $y$ direction, or as a combination of both. 


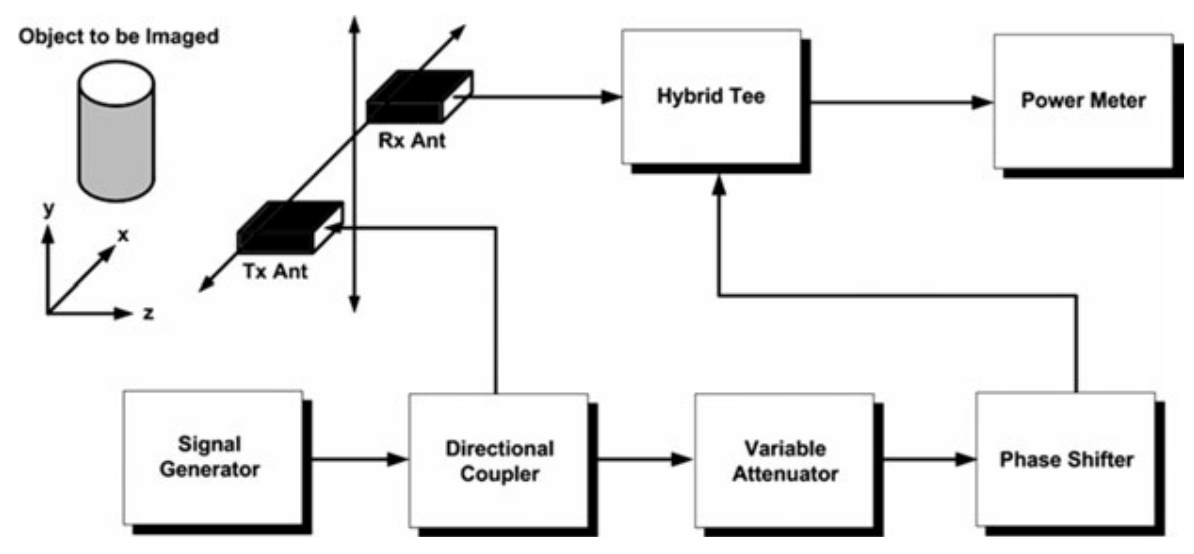

Figure 1 Outline of indirect holographic imaging system.

The process can be described without loss of generality by considering a one dimensional scan in the $\mathrm{x}$-direction. In this case the reference wave will be of the form

$$
E_{\mathrm{r}}(x)=E_{0} e^{-j k_{r} x},
$$

with

$$
k_{x}=\frac{\Delta \phi}{\Delta x}
$$

After illumination, the recorded signal cattered from the object will be of the form $E_{s}(x)$ and the intensity of the output signal from the sum port of the hybrid tee will be

$$
\begin{aligned}
I(x)=\left|E_{\mathrm{s}}(x)+E_{\mathrm{r}}(x)\right|^{2}\left|E_{\mathrm{s}}(x)\right|^{2}+\left|E_{\mathrm{r}}(x)\right|^{2} \\
=+E_{\mathrm{s}}^{*}(x) \cdot E_{\mathrm{r}}(x)+E_{\mathrm{s}}(x) \cdot E_{\mathrm{r}}^{*}(x)
\end{aligned}
$$

Taking the Fourier Transform of this expression we obtain

$$
\begin{aligned}
F\{I(x)\}= & F\left\{\left|E_{\mathrm{s}}(x)\right|^{2}\right\}+F\left\{\left|E_{0}\right|^{2}\right\}+F\left\{E_{\mathrm{s}}^{*}(x)\right\} \\
& \otimes F\left\{E_{0} e^{-k_{x} x}\right\}+F\left\{E_{\mathrm{s}}(x)\right\} \otimes F\left\{E_{0} e^{+j k_{x} x}\right\}
\end{aligned}
$$

Provided that the scattered signal can be band limited, $k<$ $k_{\mathrm{M}}$, and subject to being able to apply a suitable phase gradient to the offset reference signal, $k_{\mathrm{x}}>3 k_{\mathrm{M}}$, the third and fourth terms of Eq. (3) can be separated and the unwanted terms filtered off. If we restrict attention to the fourth term, this will produce a filtered pattern of the form

$$
F^{\prime}\{I(x)\}=F\{E(x)\} \otimes F\left\{E_{0} e^{+j k_{x} x}\right\} .
$$

Centralizing this pattern and taking the Inverse Fourier Transform gives

$$
\begin{aligned}
E^{\prime}(x) & =F^{-1}[F\{E(x)\}] \\
& =E(x)=E_{0 \mathrm{~s}} e^{-j \theta x}
\end{aligned}
$$

which is the original complex scattered field at the measurement plane.

From a knowledge of the complex field at the measurement plane, images of the scattering object can be obtained following similar back-propagation techniques as described by Sheen et al. [13].

The first two terms of this expression are centered around the origin in the Fourier domain with the third and fourth terms displaced from the origin by $\pm k_{x}$ respectively. This is shown diagrammatically in Figure 2.

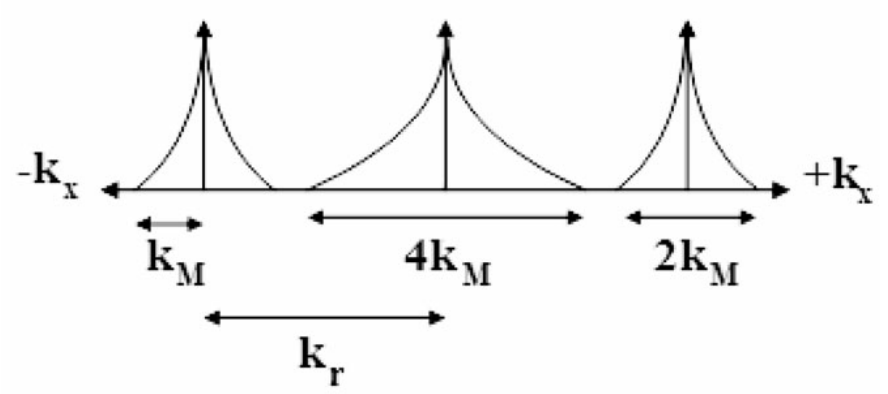

Figure 2 Spatial frequency spectrum of intensity pattern. 


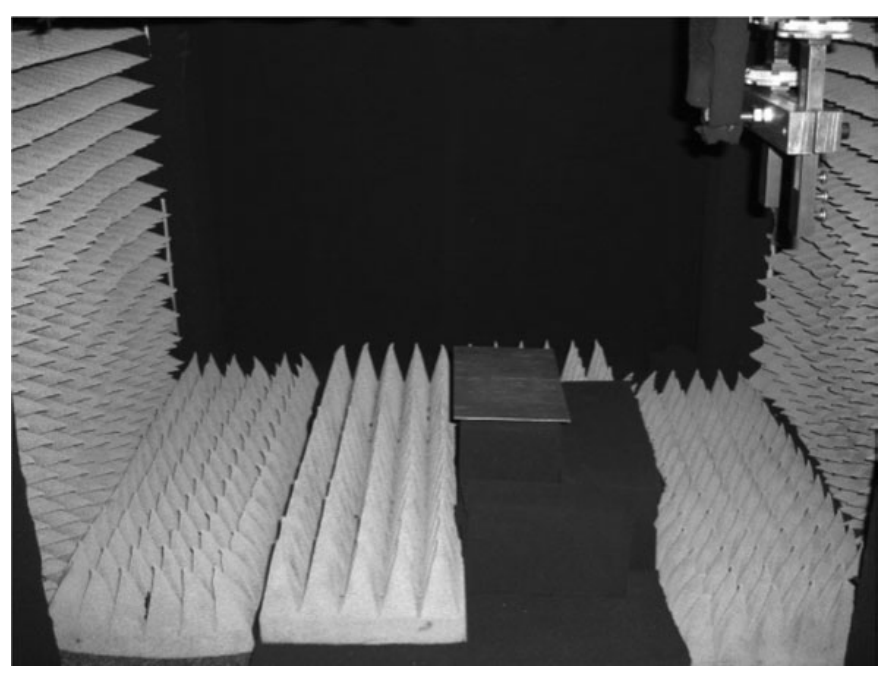

Figure 3 Holographic imaging of metal plate.

$$
\begin{array}{r}
E^{\prime}(x, z=-d)=\frac{1}{2 \cdot \pi} \int[F\{E(x)\}] \cdot e^{j \cdot k_{z} \cdot d} \cdot e^{-j \cdot k_{x} x} \cdot d x=E(x) \\
=E_{0 \mathrm{~s}} e^{-j \theta x}
\end{array}
$$

Whilst the technique of off-axis indirect holography has found widespread use at optical frequencies, its use at microwave frequencies has been limited due to practical difficulties in producing the required radiated reference signal with linear phase gradient. A method of overcoming this difficulty by using a synthesized reference signal has previously been described for the determination of antenna radiation characteristics [1-5]. This work describes how indirect holographic techniques can be extended to provide complex scattered fields of passive objects and to reconstruct images of the original object. It aims to demonstrate the ability of indirect holography to provide images of objects with dimensions smaller than one half wavelength.

\section{IMAGING OF UNCONCEALED OBJECTS}

Initial tests were conducted on a thin rectangular aluminum sheet, $200 \mathrm{~mm} \times 120 \mathrm{~mm}$, located $120 \mathrm{~mm}$ from the scanning aperture as shown in Figure 3, and at a frequency of $12.5 \mathrm{GHz}$. Results were taken at sample

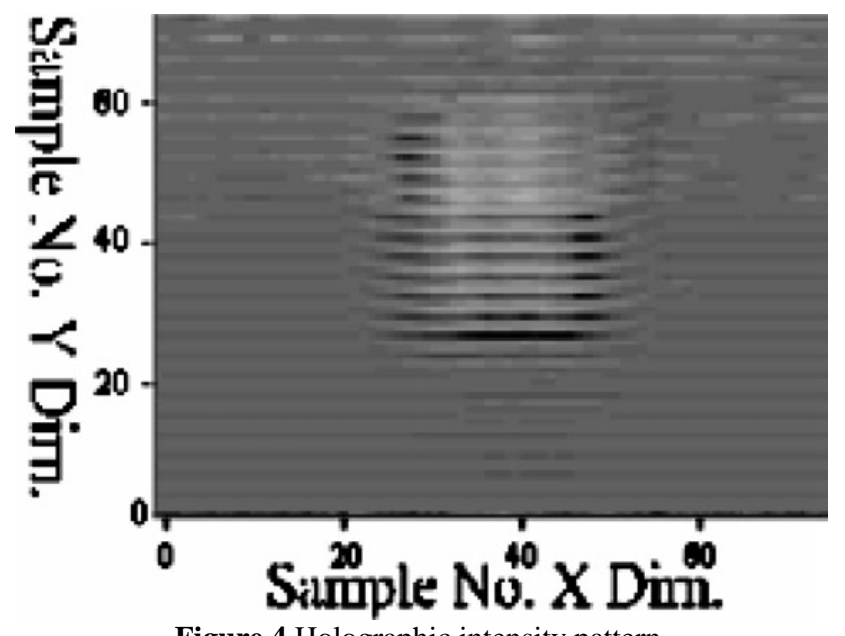

Figure 4 Holographic intensity pattern.

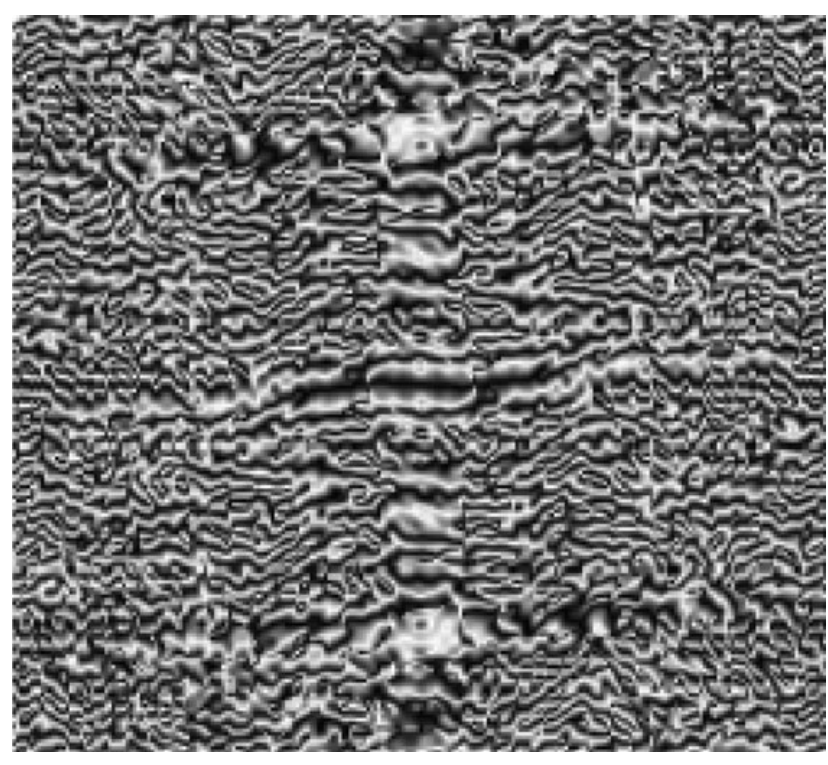

Figure 5 Fourier transform of intensity pattern.

spacing, $\Delta x=\Delta y=6 \mathrm{~mm}$ over a rectangular scanning aperture, $450 \mathrm{~mm} \mathrm{x} 450 \mathrm{~mm}$. The required offset reference signal was synthesized by introducing a phase shift, $\Delta \Phi=2 \pi / 3$ rads, between sample spacing, $\Delta x=6$ $\mathrm{mm}$ producing an offset reference wave vector, $k r=349$ $\mathrm{rads} / \mathrm{m}\left(\mathrm{k}_{0}=261.8 \mathrm{rads} / \mathrm{m}\right)$.

Results in Figure 4 show the recorded holographic intensity pattern over a $450 \mathrm{~mm}$ x $450 \mathrm{~mm}$ scanning aperture with a linear phase shift applied along the horizontal axis with horizontal and vertical axes are shown in terms of the sample number.

The experimental results have been zero filled to a $256 \mathrm{x}$ 256 data array before being transformed into the Fourier Domain to provide results in the frequency domain as shown in Figure 5.

Following the procedure outlined in the previous section and Eqs. (4)-(6), the complex scattered field at the measurement plane can be reconstructed as shown in Figure 6. Examination of these results show good correspondence with the original object over the upper half of the image but poor correspondence over the lower half.

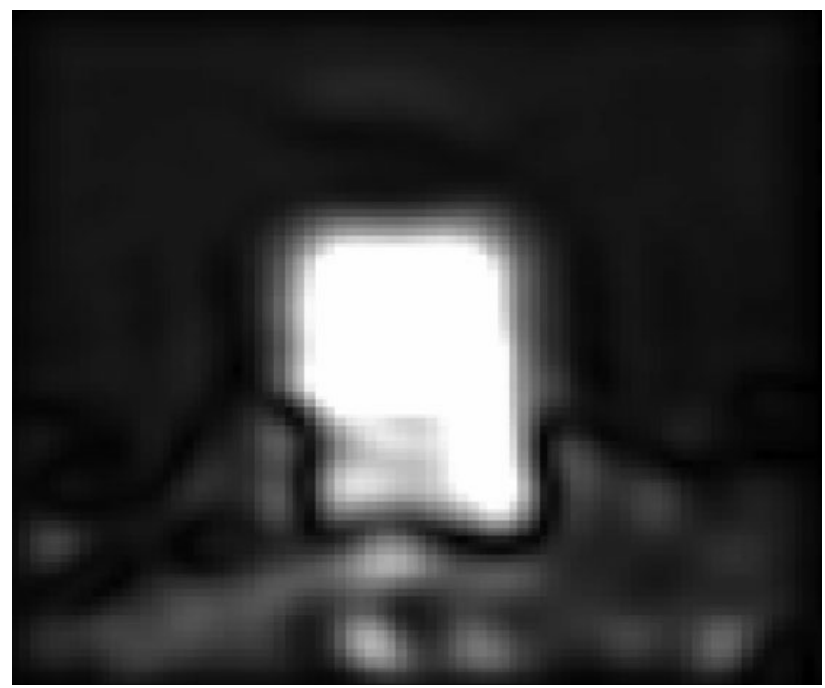

Figure 6 Reconstructed image of metal sheet. 


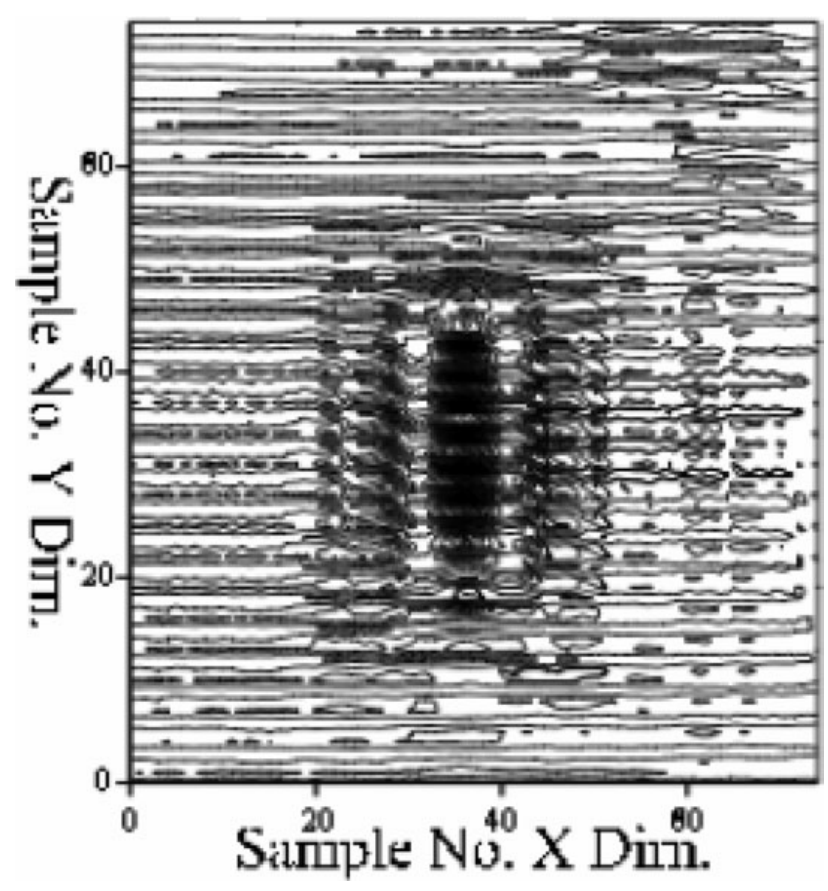

Figure 7 Measured intensity pattern for single metal plate.

Results for the reconstructed phase image also provided poor correspondence with the original object.

Improvements were made to the shielding surrounding the scanner aperture and further results were taken on a small rectangular aluminum plate of dimensions, 130 x $50 \mathrm{~mm}$, located at a distance, $z=120 \mathrm{~mm}$ from the antennas.

The measured holographic intensity pattern is shown in Figure 7.

Following the procedure outlined above, reconstructed images of the original plate were obtained at the position of the measurement plane. Results in Figure 8 show reconstructed phase and magnitude patterns from indirect

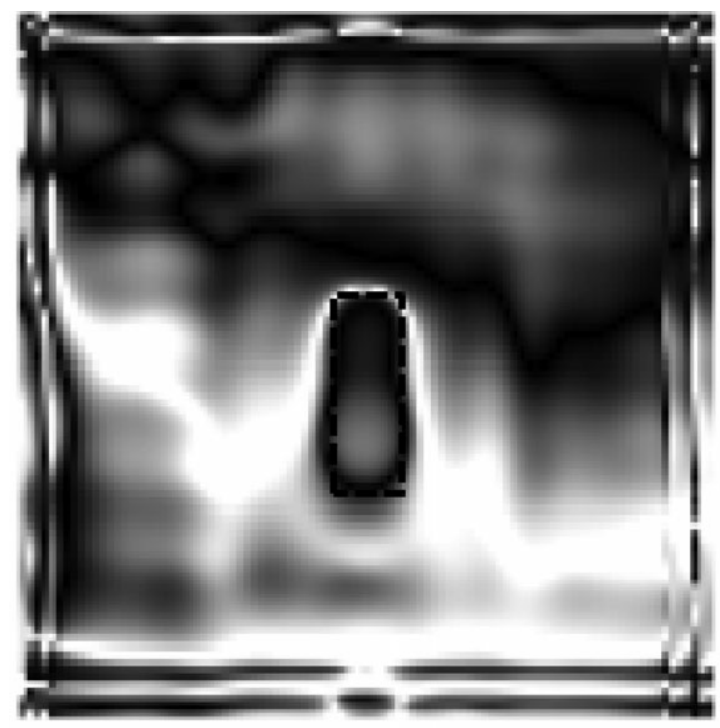

(a) Reconstructed phase image

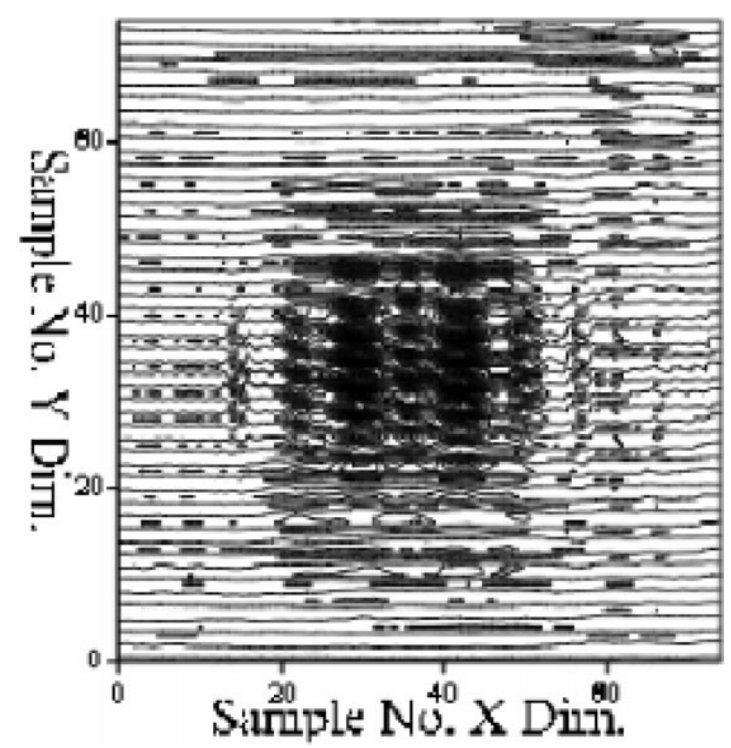

Figure 8 Measured holographic pattern for single plate.

holographic results. The introduced dashed rectangular pattern in Figure 8a demonstrates the outline of the actual plate for comparison.

It can be seen that good agreement is obtained for both phase and magnitude images.

To investigate the ability of this technique to discriminate between closely spaced objects, two small aluminum plates, each $90 \mathrm{~mm} \times 50 \mathrm{~mm}$ with a separation of $20 \mathrm{~mm}$, were imaged. The resultant holographic intensity pattern is shown in Figure 9.

The reconstructed phase and magnitude images are shown in Figure 10.

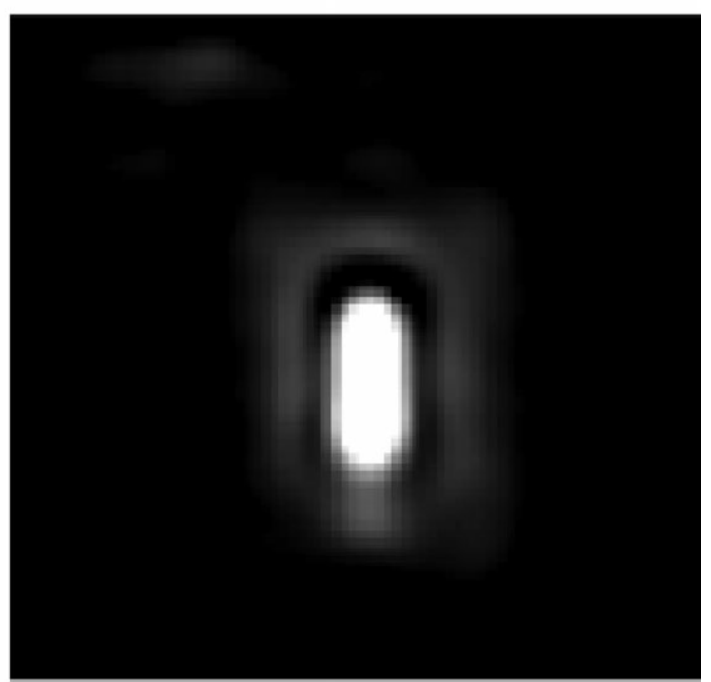

(b) Reconstructed magnitude image

Figure 9 Reconstructed images of single metal plate. (a) Reconstructed phase image, (b) reconstructed magnitude image. 


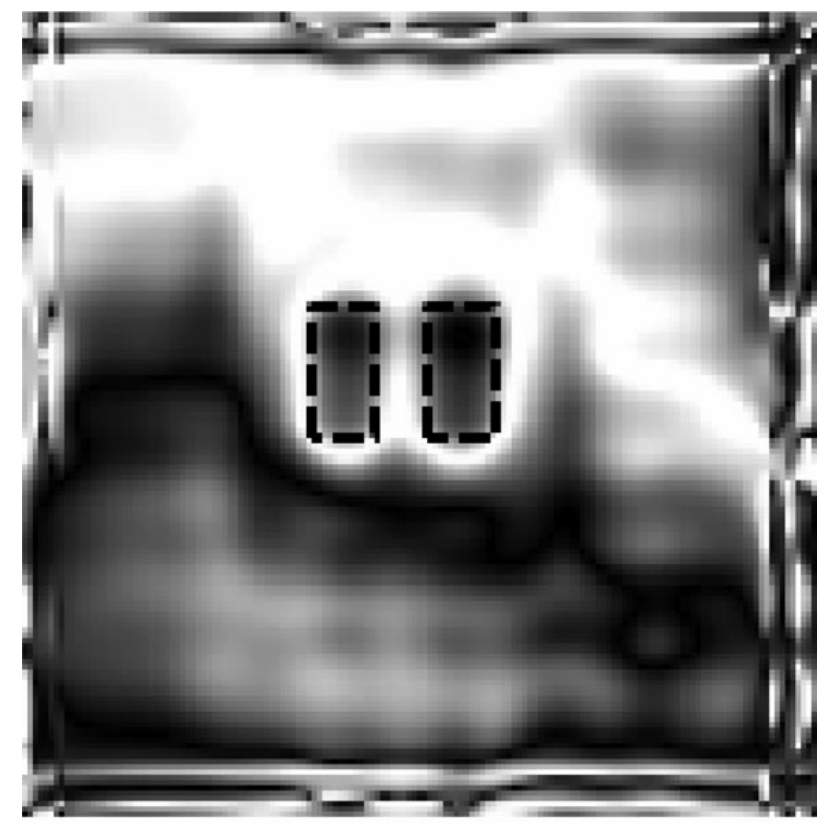

(a) Reconstructed phase image

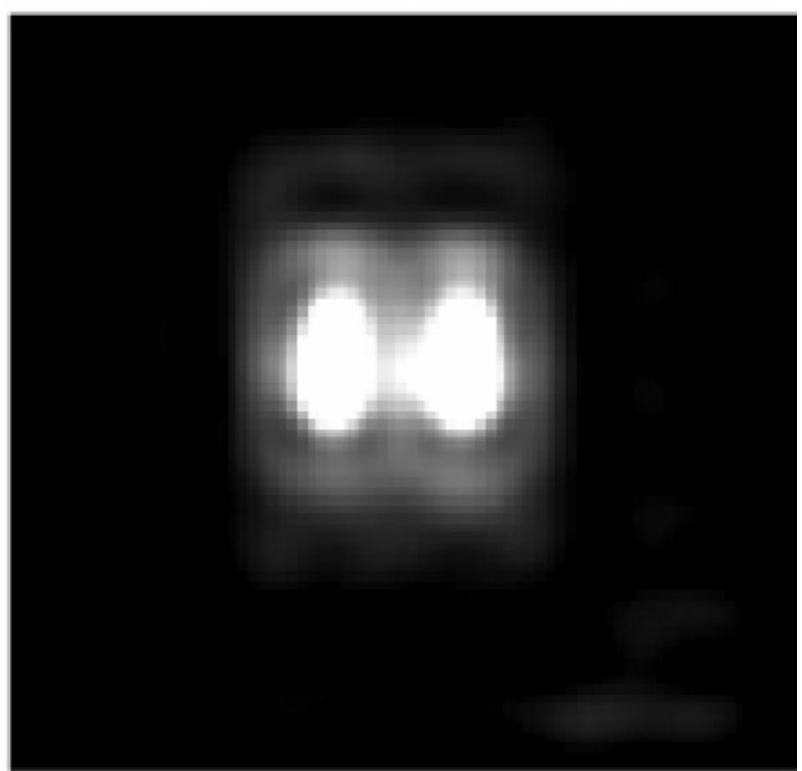

(b) Reconstructed magnitude image

Figure 10 Reconstructed images of two aluminum plates. (a) Reconstructed phase image, (b) reconstructed magnitude image

Whilst it may be difficult to discern whether the original object consisted of a single plate or two plates from the magnitude image, the presence of two plates, together with their sizes and separation is clearly evident from the reconstructed phase image.

\section{IMAGING OF CONCEALED OBJECTS}

Further tests were undertaken to investigate whether this technique could be used to image concealed objects successfully. In this case the test object consisted of a small aluminum plate, $100 \mathrm{~mm}$ x $60 \mathrm{~mm}$, attached to the underside of an $8 \mathrm{~mm}$ thick sheet of polythene of dimensions, $240 \mathrm{~mm}$ x $240 \mathrm{~mm}$. A view of the test object with the metal side uppermost is shown in Figure 11a.

$450 \mathrm{~mm} \times 450 \mathrm{~mm}$, at a frequency of $12.5 \mathrm{GHz}$ with similar sample spacing and reference phase offset.

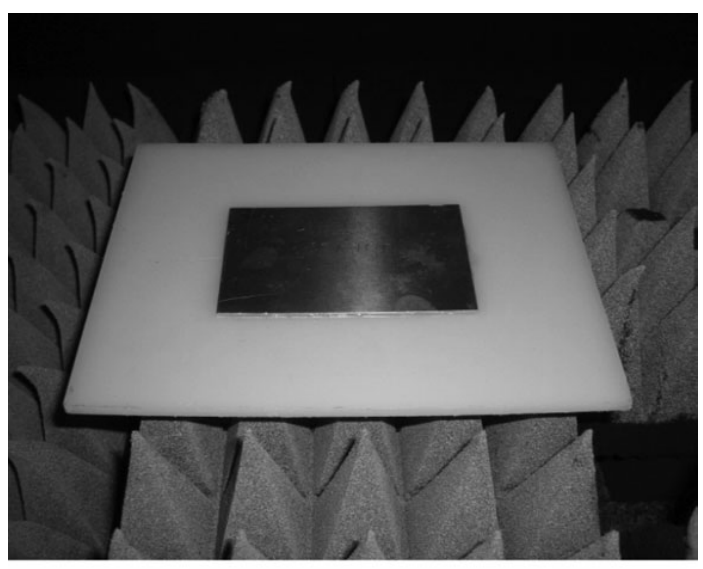

(a) Small metal plate attached to dielectric sheet

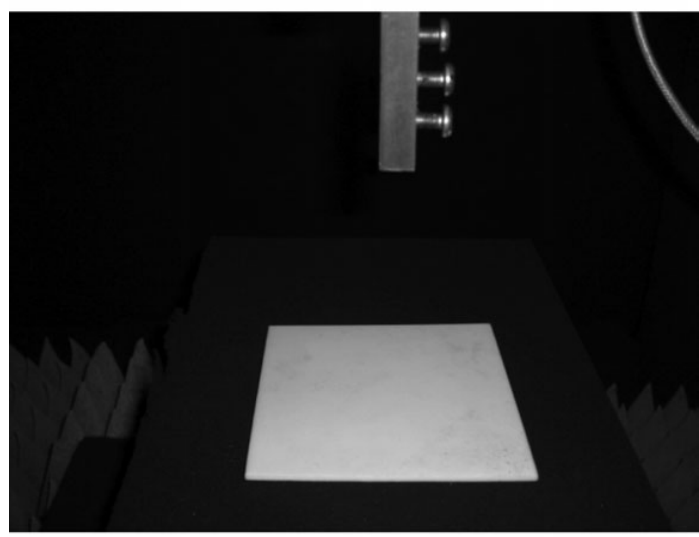

(b) Plate arrangement for holographic imaging

Figure 11 Imaging of concealed metal plate. (a) Small metal plate attached to dielectric sheet, (b) plate arrangement for holographic imaging.

The object was arranged as shown in Figure 11b with the metal plate under the plastic sheet for holographic imaging.

In a similar manner to the previous section, holographic intensity measurements were taken over a scanning aperture,

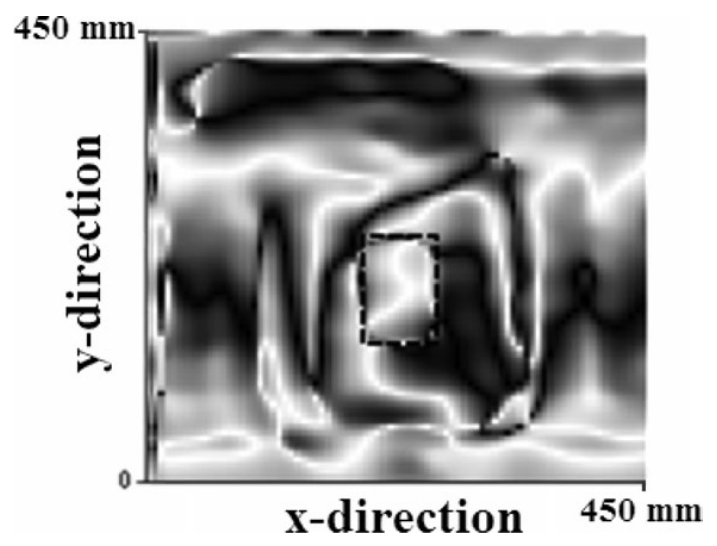

Figure 12 Reconstructed image of concealed metal plate under dielectric.

Reconstructed images at the position of the original object are shown in Figure 12. As before the horizontal and 
vertical axes represent the original physical aperture of $450 \mathrm{~mm}$ x $450 \mathrm{~mm}$.

The outline of the metal plate is clearly visible with approximate dimensions $110 \mathrm{~mm}$ x $75 \mathrm{~mm}$ together with a loosely defined boundary for the more weakly scattering dielectric cover sheet. These results compare well with the dimensions, $100 \mathrm{~mm}$ x $60 \mathrm{~mm}$, of the original object.

\section{IMAGING OF CIRCULAR OBJECTS}

In order to examine the ability to image curved objects, a test object consisting of a circular metallic annulus of diameter, $\mathrm{d}_{\mathrm{o}}=115 \mathrm{~mm}$, and internal diameter, $d \mathrm{i}=95 \mathrm{~mm}$ was used. This annulus formed the outer ring of a small corrugated horn as shown in Figure 13. At $12.5 \mathrm{GHz}$ the width of this annulus, $w=10 \mathrm{~mm}$, was less than half a wavelength $(\lambda / 2=12 \mathrm{~mm})$.

Experimental results were recorded in a similar manner to that outlined above. In this case the reconstructed image is shown in Figure 14. From Figure 14, it is possible to discern the outline of the antenna rim and provide an approximate value of diameter, $d=125 \mathrm{~mm}$, and a width value, $w=17 \mathrm{~mm}$. Also shown for comparison is the outline of the actual horn aperture. The agreement with the original outline is apparent from these results.

\section{CONCLUSIONS}

This work has provided details of a simple method for the imaging of objects using indirect holography which has been shown able to reconstruct images of objects of less than one half wavelength in dimension. By forming a holographic intensity pattern from the scattered fields and a reference signal with known linear phase shift, separation of terms can be achieved in the Fourier Domain. By extracting the required term of the Fourier pattern and performing an Inverse Fourier Transform, the original scattered fields at the measurement plane can be reconstructed. Additionally, by using back propagation

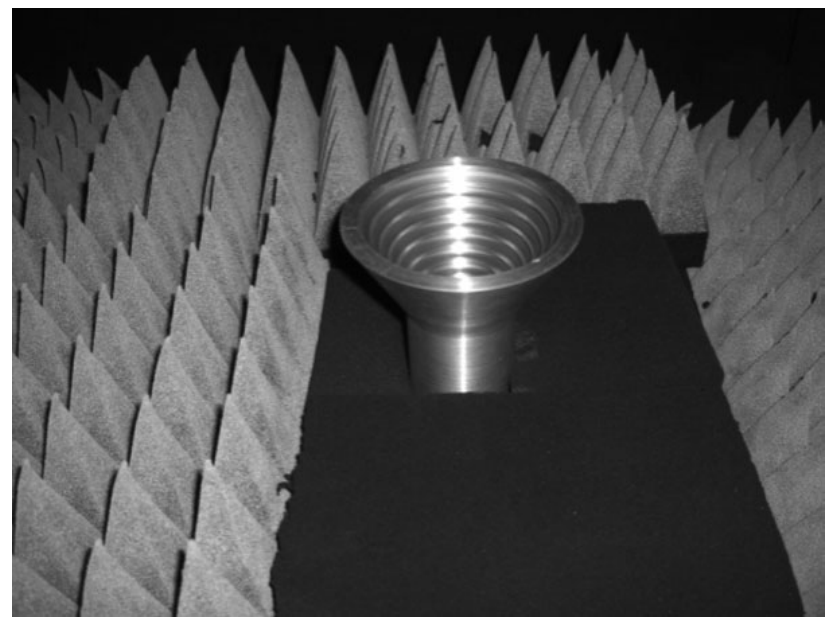

Figure 13 Imaging of conical horn aperture.

8. F. Las-Heras and T.K. Sarkar, A direct optimization approach for source reconstruction and NF-FF

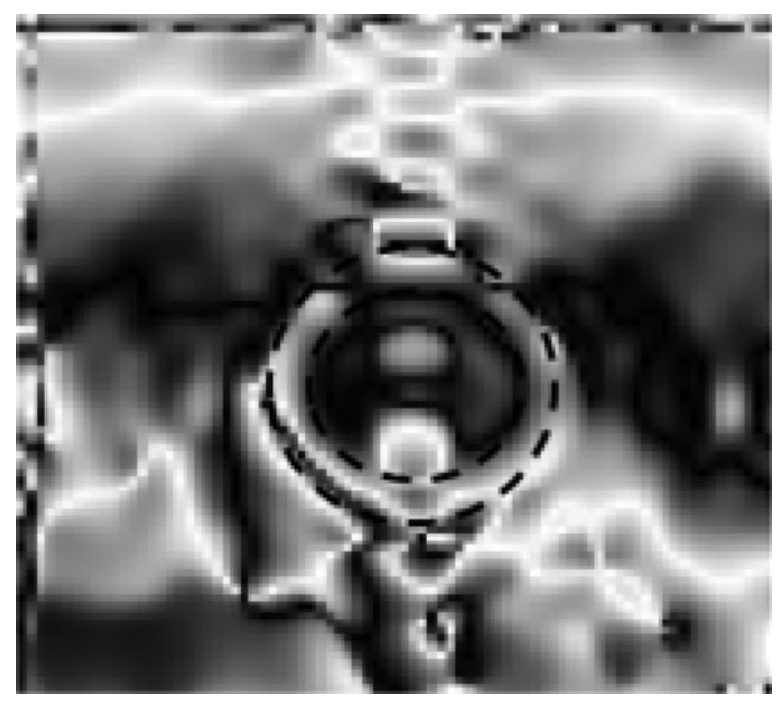

Figure 14 Reconstructed image of conical horn.

principles, the scattered fields at the position of the original object can be reconstructed. Results for a range of simple objects have shown that this technique can provide good quality images. This technique has been shown to be able to provide good images of objects with dimensions less than one half wavelength. Whilst this work has been performed for a single frequency, $f=12.5 \mathrm{GHz}$, it can be extended to multiple frequencies and to millimeter wave imaging.

\section{REFERENCES}

1. J.C Bennett, A.P. Anderson, P.A. McInnes, and A.J.T. Whitaker, Microwave holographic metrology of large reflector antennas, IEEE Trans Antennas Propagat 24 (1976), 295-302.

2. D. Smith, M. Leach, and A. Sambell, An indirect holographic method for determining antenna radiation patterns and imaging antenna fields, Proc IEEE Int Antennas Propagat Symp Dig 4 (2002), 706-709.

3. D. Smith, M. Leach, M. Elsdon, and S. J. Foti, Indirect holographic techniques for determining antenna radiation characteristics and imaging aperture fields, IEEE Antennas Propagat Mag 49 (2007), 54-67.

4. V. Schejbal, V. Kovarik, and D. Cermak, Synthesized-reference-wave holography for determining antenna radiation characteristics, IEEE Antennas Propagat Mag 50 (2008), 71-83.

5. V. Schejbal, J. Pidanic, V. Kovarik, and D. Cermak, Accuracy analyses of synthesized-reference-wave holography for determining antenna radiation characteristics, IEEE Antennas Propagat Mag 50 (2008), 89-98.

6. O. M. Bucci and C. Genarelli, Use of sampling expansions in near-field-far-field transformations; the cylindrical case, IEEE Trans Antennas Propagat 36 (1988), 830-835.

7. T. Isernia, G. Leone, and R. Pierri, Radiation pattern evaluation from near-field intensities on planes, IEEE Trans Antennas Propagat 44 (1996), 701-710. transformation using amplitude only data, IEEE Trans Antennas Propagat 50 (2002), 500-510. 
9. C.H. Schmidt, S.F. Razavi, T.F. Eibert, and Y. Rahmat-Samii, Phaseless spherical near-field antenna measurements for low and medium gain antennas, Adv Radio Sci 8 (2010), 43-48.

10. R. Pierri, G. D'Elia, and F. Soldovieri, A two probes scanning phaseless near-field far-field transformation technique, IEEE Trans Antennas Propagat 47 (1999), 792-802.

11. Y.S. El-Said and A.M. Attiya, Modified two-probe approach for amplitude-only near-field measurements, Microwave Opt Technol Lett 41 (2004), 270-273.
12. S. Costanzo, G. D’Massa, and M.D. Migliore, A novel hybrid approach for far-field characterization from near-field amplitude-only measurements on arbitrary scanning surfaces, IEEE Trans Antennas Propagat 53 (2005), 1866-1873.

13. D.M. Sheen, D.L. McMakin and T.E. Hall, Threedimensional millimetre wave imaging for concealed weapons detection, IEEE Trans Microwave Theory Tech 49 (2001), 1581-1592.

\section{BIOGRAPHIES}

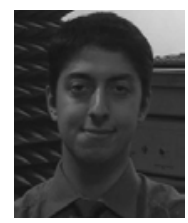

Okan Yurduseven received his BSc and MSc in Electronics and Communications Engineering on Microwaves/Antennas and Propagation from Yildiz Technical University, Istanbul, Turkey with first-class honors degrees in 2009 and 2011, respectively. He is currently a PhD Researcher with a full PhD scholarship in the School of Computing, Engineering, and Information Sciences at Northumbria University in Newcastle upon Tyne, United Kingdom where he also works as a parttime Lecturer. He received the best student paper award at the 2012 Mediterranean Microwave Symposium (MMS) in Turkey for the work on Indirect Microwave Holography conducted under the supervision of Prof. David Smith. His research interests include microwave imaging, multiband/wideband printed antennas, novel designs of X-band reflector and feed horn antennas for advanced air/coastal surveillance radar systems, antenna measurement techniques and radar cross section analysis. He is the author/coauthor of more than 20 refereed publications.

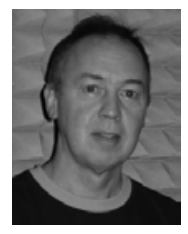

David Smith received his BSc degree in Physics and $\mathrm{PhD}$ in Electrical and Electronic Engineering from Newcastle University in 1972 and 1987, respectively. He has been a member of the IEEE for over 30 years. He is currently Reader in Microwave Engineering at Northumbria University, a post he has held since 2004. Prior to this, he was Head of Electrical and Electronic Engineering Division at Northumbria University. He has acted as a consultant to a number of companies, including Andrew Corporation, Matra-Marconi, and 3M. He is the author of over 40 technical publications and patents. His current research interests include satellite antenna design, antenna measurement techniques, indirect microwave holographic imaging, and material measurement using open resonators.

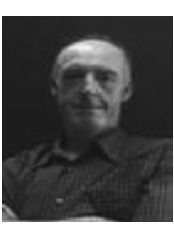

Bryan Livingstone received his BSc and $\mathrm{PhD}$ degrees in Electrical and Electronic Engineering from Newcastle University in 1976 and 1981, respectively. He was a Research Associate at Newcastle University between 1979 and 1982 researching into $\mathrm{NiCr}$ Thin Film Resistors and was the Chairman of the Analogue and Digital Designs

Ltd between 1987and1992. He has been working as a Lecturer at Northumbria University since 1982. He is currently the Program Leader of the BEng $(\mathrm{H})$ Electrical Electronic Engineering, MSc Optoelectronic and Communications Engineering, and MSc Microelectronic and Communications Engineering. His research interests include analogue electronics and instrumentation, RF, microwave and digital communication systems, communication networks and digital signal processing.

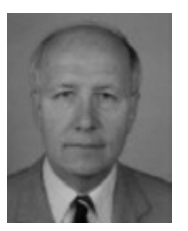

Vladimir Schejbal graduated from the Czech Technical University, Prague, in 1970. He received the $\mathrm{PhD}$ degree from the Slovak Academy of Science, Bratislava, in 1980. He was with the Radio Research Institute Opocinek, the Czech Republic (Antenna Department) from 1969 to 1993. From 1983 to 1986, he was on leave with the Higher Institute of Electronics (Microwave Department) Beni Walid, Libya, as a lecturer. He has been with the University of Pardubice, the Czech Republic, since 1994, now as a full professor and a head of the department. He is interested in microwave antennas and propagation. He has published over

100 papers. He is a Senior Member of the IEEE.

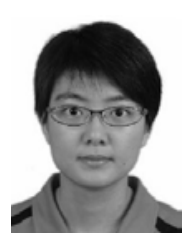

Zhonghui You was born in Jiangsu, China, in 1988. She received her B.Eng(Hons) degree in Mobile Communication Engineering from Northumbria University and B.Eng degree in Mobile Communication Engineering from Nanjing University of Posts and Telecommunications in 2011. She is a postgraduate student in MSc Microelectronic and Communication Engineering. 